\title{
Dissolution: a Continuing Perspective
}

\author{
Roger L. Williams ${ }^{1}$ and Thomas S. Foster ${ }^{2}$ \\ ${ }^{1}$ Chair, Council of Experts \\ ${ }^{2}$ Chair, Biopharmaceutics Expert Committee \\ United States Pharmacopeia, Rockville, MD
}

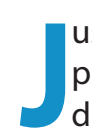

ust short of five years ago ${ }^{1}$ Dissolution Technologies

published an article that provided a regulatory view of

dissolution testing and its role vis-à-vis bioavailability

(BA) and bioequivalence (BE). That article emphasized reg-

ulatory progress about the characterization and/or perfor-

mance tests that help assure product quality, safety and

efficacy. That progress built on the substantial work of the past, beginning with the Office Technology Assessment report in the 1970s, the follow-on 1977 Food and Drug Administration (FDA) regulations at 212 CFR 320, and, perhaps most importantly, the Drug Price Competition and Patent Term Restoration Act in 1984. This Act established a fully operational approach to allow interchangeable innovator and generic products in the US. In the 1990s, International Conference on Harmonization (ICH) and World Health Organization (WHO) created important and complementary documents in the area of product quality, with $\mathrm{ICH}$ focusing primarily on guidances supporting a regulatory filing for first-entry products and WHO working to promote good quality, interchangeable, multisource products. ${ }^{2,3}$ In parallel, the FDA created a series of guidances and associated approaches that were intended to be more detailed 'how-to' documents, amplifying the science understanding of the 1977 regulations. In retrospect, the early work of the 1960s and 1970s, as expressed in these regulations, has stood the test of time, and the unacknowledged authors of these regulations, many of whom are still with us today, should be recognized. While the FDA guidances of the 1990s extended the science of the 1977 regulations, much remains to be done. Despite the early promise of the biopharmaceutics classification system, it does not appear at the present time to have been taken up vigorously by either innovator or generic manufacturers as a means of reducing regulatory and/or clinical trial burden. Several key BA/BE guidances, particularly for locally acting drugs, are unfinished. And while the FDA's solution to the problem of switchability of the 1990s (the widely commented on individual bioequivalence criterion) failed, just as did the earlier '75-75' approach, the challenges of assuring switchability (which the average criterion does not address well), of documenting bioequivalence for highly variable drugs, and of addressing the public health concern of subject by formulation interactions for certain dosage forms, still remain.

Turning to compendial approaches, it is perhaps unsurprising that, while much progress has been achieved in the last several years, much work also remains to be done. This work has begun in several key areas. First, USP has considered the underlying science and technical issues related to the compendial performance test. These considerations build readily on the FDA, ICH, and WHO approaches. BA and BE studies conform to the general approaches delineated in the $\mathrm{ICH}$ Q6A document, i.e., they are characterization studies that establish, for purposes of a regulatory filing, the performance of a specific drug product. In this context, disintegration or dissolution tests with acceptance criteria, which are specifically mentioned in ICH Q6A, may become one of a series of tests in the drug product specification. In the absence of an in vitro/in vivo correlation, which is the case for most dosage forms, the BE characterization test is more determinative than an in vitro dissolution or disintegration batch release test, i.e., bioequivalent drug products may still rely on different dissolution or disintegration procedures and acceptance criteria to assess performance. This is generally expressed in the phrase 'the in vivo study trumps dissolution' and supports a flexible USP dosage form monograph with different dissolution 'cases,' so long as the primary documentation of BA/BE remains intact. Evidence in the literature also suggests that dissolution usually is more sensitive than an in vivo $\mathrm{BA} / \mathrm{BE}$ study. This is gratifying in that dissolution thus will usually be an early warning signal of a problem in dosage form performance. Over time, the private dissolution or disintegration test may become the public performance test in the United States Pharmacopeia (USP). Both the private and public approaches frequently rely on suitably validated and calibrated disintegration and dissolution approaches, which can be developed using both the FDA guidances and USP General Chapters. Determinations about $\mathrm{BA}$ and $\mathrm{BE}$ are the responsibility of FDA and do not involve USP. Further, the USP Performance test is related to BA and BE only when closely allied with a sound regulatory determination and with strict post-approval change control. These are expressed in SUPAC and subsequent ('make your own SUPAC') guidances from FDA. Without this link and assurance, the USP Performance test should be regarded solely as a quality control test for non-compliance and/or batch release.

Based on the general science and technical understanding that links BA, BE, and dissolution, USP has submitted an overview article for publication that speaks to the study design and analysis aspects of dissolution. This attempts to clarify the hypothesis testing component of the dissolution procedure; suggests a testing by variables as opposed to testing by attributes (parametric versus non-parametric testing); posits a tolerance interval approach as a means of setting acceptance criteria; and supports more flexible study designs. The general discussion in the paper recommends a more clear delineation of consumer and producer (manufacturer) risk in support of clear communications about risk assessment, management, and communication. USP is also working to expand 
the availability of the USP Performance test, moving beyond the dissolution procedure for orally administered non-solution dosage forms, to procedures for dosage forms given by injection and by topical, inhalation, and mucosal routes of administration. This will be a protracted effort, involving many stakeholders, with the end goal being a fully functional set of USP Performance tests for all dosage forms. Associated with this work is a parallel effort on dissolution calibrators. USP has acknowledged that mechanical calibration is suitable for many parameters that can affect the results of a dissolution procedure. There are still several parameters, however, that are not adequately assessed by mechanical calibration. Through two Project Teams, \#5 Dissolution Calibrators and \#6 Biopharmaceutics, under USP's Prescription/Non-Prescription Drug Stakeholder Forum, USP has enhanced its collaborative work with manufacturers. These manufacturers will be highly important in the development of better calibrators for USP, not only for currently available USP Performance tests but also for the new Performance tests that may emerge. The overall output of this work will be expressed in new USP General Chapters and in revisions to current ones. For example, USP is now involved in revision of General Chapter In Vivo Bioequivalence Guidances $<1090>$ to bring it more in line with FDA guidances and with $\mathrm{WHO}$ and ICH documents. In addition, harmonization in the Pharmacopeial Discussion Group, composed of representatives from the Japanese (JP), European (EP), and US pharmacopeias (USP), with WHO as an observer, has completed work that harmonizes currently available disintegration and dissolution procedures.

Where does the future lie? It is difficult to predict, but certainly $\mathrm{BA}, \mathrm{BE}$, and dissolution will become increasingly important in the years to come as world manufacturers advance quality approaches for ingredients and dosage forms. Many countries, including Brazil and Mexico in the Americas, are joining the US and Canada in evolving a system of interchangeable innovator and generic dosage forms. The possibility of a single comparator pharmaceutical product, i.e., reference listed drug, for the Americas has been broached in the BA/BE Working Group for the Pan American Network for Drug Regulatory Harmonization. And the FDA's process analytical technology approach is considering how better process understanding can allow both more variability in manufacturing steps as well as reduced end product testing. This new approach is especially challenging, given that the goal is not to reduce end product testing per se but rather, for dissolution testing, to assure comparable in vivo performance before and after a manufacturing change.

In sum, the last forty years have witnessed remarkable progress in developing ways to assess product performance and to assure that this performance is stable over time. The general task is a considerable one and is critical to a successful system of innovator and interchangeable multisource products. The burden falls to both innovator and generic companies. Innovators must assure continuing equivalence in the presence of post-approval change in order for the system to work. Generic manufacturers must also assure this equivalence at the time of market entry and with postapproval change as well. While much has been done, much remains to be done, not only nationally but internationally with the end goal the availability of good quality, interchangeable dosage forms for practitioners and patients.

\section{References}

1 Shah VP, Williams RL: Role of Dissolution Testing in Regulating Pharmaceuticals, Dissolution Technologies, 1999 6(3), 7.

2 Multisource (generic) pharmaceutical products: guidelines on registration requirements to establish interchangeability (WHO Expert Committee on Specifications for Pharmaceutical Preparations, ThirtyFourth Report, 1999)

3 Guidance on the selection of comparator pharmaceutical products for equivalence assessment of interchangeable multisource (generic) products, $\mathrm{WHO}$ Expert Committee on Specifications for Pharmaceutical Preparations, Thirty-Sixth Report, 1999. 


\title{
Future Directions for Academic Research in Dissolution Testing
}

\author{
Jennifer B. Dressman \\ Department of Pharmaceutical Technology, J. W. Goethe University, \\ Marie-Curie Str. 9, 60439 Frankfurt am Main, Germany
}

email:Dressman@em.uni-frankfurt.de

D issolution testing has enjoyed a resurgence of interest in the last decade, not only from a regulatory point of view, but also in the academic setting. This can be partly attributed to the widespread acceptance of the Biopharmaceutics Classification Scheme, and partly due to the advent of new technologies employed in test procedures.

The Biopharmaceutics Classification (BCS) scheme provides for dissolution testing as a surrogate for pharmacokinetic studies to establish therapeutic equivalence of two drug products. Currently its scope is limited to oral drug products containing drugs belonging to Class I (highly soluble, highly permeable) and which can release the drug rapidly. These stringent requirements, invoked by the FDA for safety reasons, have resulted in meager implementation of the biowaiver. The parameters for the biowaiver are now being challenged on all three fronts. Should we allow biowaivers for drugs belonging to Class II but which are highly soluble at intestinal $\mathrm{pH}$ ? Should we allow biowaivers for substances belonging to Class III? Should the dissolution criterion be relaxed to $85 \%$ in 45 minutes from the current 30 minutes? As more relevant papers (pro and con) appear in the literature we will be better positioned to reevaluate the parameters.

A further limitation to application of the BCS has been the lack of a useful data bank in the literature with the appropriate classifications. Here also, academic efforts are being directed to classify drugs either by combinations of in silico predictions and literature data (Amidon et al., Molecular Pharmaceutics, 2004, 1:85-96) or literature data coupled with experimental data (Lindenberg et al., in press Europ.J. Pharm. Biopharm. 2004). A further initiative by Dirk Barends of the RIVM in the Netherlands and his colleagues is to produce a series of comprehensive monographs of individual substances containing information relevant to BCS classification (see website and newsletter of the Biopharmaceutics working group under http://www.fip.org/bps/science_bioequivalence.htm). So it seems that within the next five years a comprehensive data base on drug classification according to the BCS will exist.

The discussion about quality control versus biorelevant testing continues. Ideally, a dissolution test should reflect in vivo performance, no matter whether it is being used for quality control, to argue for a biowaiver or to establish an in vitro-in vivo correlation (IVIVC). However, for compounds belonging to Class III and IV of the BCS, possibilities for predicting in vivo performance from dissolution data are limited because the permeability of the compound is at least partly limited by a parameter that is not addressed in the dissolution test, namely the permeability. Although some Class III drugs might be suitable for biowaivers (see above), this will be unlikely the case for Class IV drugs and application of dissolution testing for products containing these is likely to be limited to quality control. For other compounds, dissolution test design is being optimized to better reflect physiological conditions in the Gl tract while retaining the simplicity that has traditionally been so attractive in the dissolution methodology.

One key area in which academic research has been focussed recently is to improve our understanding of the hydrodynamics in the Gl tract as well as in the dissolution apparatus. In Prof. Owen Corrigan's research group at Trinity College in Dublin the focus is on modelling of the hydrodynamics in the dissolution apparatus, while groups in the USA (Prof. Brasseur at Penn State) and Germany (Prof. Weitchies at the University of Greifswald) are creating better models of fluid and solid movement in the stomach. There have also been several attempts to correlate stirring rates in vitro with those in dogs, principally by European and Japanese research groups. I am confident that within the next decade we will have a much clearer understanding of how to relate hydrodynamic conditions in the Gl tract to those we use in the laboratory and that this will result in better IVIVC.

A second area of activity in academic research has been to shift the focus of biorelevant testing from immediate release to controlled release dosage forms, especially to predict food effects on dosage form performance and also to indicate interchangeability of drug products. Media have been designed to mimic conditions throughout the Gl tract (most recently by Klein et al., J. Pharm. Pharmacol. 56:605-610 (2004)) and have been applied to date mainly in the Reciprocating cylinder (USP Type III) and Flow through (USP Type IV) apparatus. The goal is to develop biorelevant dissolution test methodology that will facilitate in vitro-in vivo correlation and thereby reduce time-to-market for new $C R$ drug products.

A third area of focus has been the evaluation of fiber optic methodology for in situ analysis of drug concentration in the dissolution vessel. This methodology has taken a solid foothold, especially for quality control purposes where the methodology facilitates a high throughput due to the relative ease of automating the test. The initial concerns that excipients would interfere with the detection of the drug have proven to be unfounded in many cases, although this must always be checked on a formulation by formulation basis. 
Another area of continued interest is the development of dissolution tests for "special" dosage forms - special in the sense that they are not standard orally administered tablets and capsules. There has been an FIP initiative in this are, which resulted in a white paper and several workshops in both Europe and the USA on the topic. Especially interesting are tests to evaluate so-called "flash-tabs", orally disintegrating dosage forms, and the use of accelerated test procedures for dosage forms which release drug over very long periods (e.g. implants, stents, depot injections).

What is the outlook for dissolution testing? In the next years, we can expect to see increasing numbers of drug products approved on the basis of dissolution tests as a means to demonstrate therapeutic equivalence, more rational design of dissolution tests for controlled release as well as immediate release drug products, and a reduction in time to market through biorelevant screening of formulations and application of in vitro-in vivo correlations. Further, it will be interesting to see how the relationship between near-IR methodology and dissolution testing evolves in terms of quality assurance and control.

\section{Literature}

1. Kasim NA, Whitehouse M, Ramachandran C, Bermejo $M$, Lennernäs $H$, Hussain AS, Junginger HE, Stavchansky SA, Midha KK, Shah VP, Amidon GL. Molecular Properties of WHO Essential Drugs and Provisional Biopharmaceutical Classification. Molecular Pharmaceutics, 2004, 1:85-96.

2. Dressman JB, Lindenberg M. Classification of Orally Administered Drugs on the WHO Model List of Essential Medicines According to the Biopharmaceutical Classification System (BCS). Europ. J. Pharm. Biopharm. (in press, 2004).

3. S. Klein, J. Butler, J. Hempenstall, C. Reppas, J Dressman Media to simulate the postprandial stomach. I. Matching the physicochemical characteristics of standard breakfasts. J. Pharm. Pharmacol. 56: 605-610 (2004) 


\title{
Role of Dissolution Testing: Regulatory Perspectives
}

\author{
Vinod P. Shah, Ph. D. \\ Office of Pharmaceutical Science, Center for Drug Evaluation and Research \\ Food and Drug Administration, Rockville, MD
}

0 ver the last three decades, the dissolution test has come a long way and has evolved into a powerful tool for characterizing oral drug product and their in vivo performance. It is an important tool in drug development and in quality control. In drug development, it is used to guide formulation development and to select appropriate formulations for in vivo testing. In quality control, it is used as a batch-to-batch quality test before the product is released in the market. This has been possible only because of our increased knowledge and understanding of science behind the test methodology, and continuous improvement of the test equipment. The dissolution test procedure is well established, reliable and reproducible and has brought about changes in regulatory perspectives.

The dissolution test, once only a traditional quality control test, has now become also a surrogate in vitro bioequivalence test. This represents a shift in dissolution perspective. A new regulatory perspective has emerged as evidence from biowaiver criteria based on dissolution profile comparison. Several regulatory guidances have been developed which refer to 'dissolution', and provide biowaivers based on dissolution profile comparison, e.g., Biopharmaceutics Classification System and General Bioavailability/Bioequivalence guidance. In addition, several SUPAC (Scale-up and Post-Approval Changes) guidances are published which rely on dissolution or in vitro release test for assuring product sameness after certain manufacturing and/or formulation changes.

The utility of the dissolution test may be greatest in developing countries, where it can be used as an in vitro bioequivalence test. One important application of the dissolution test is its value in assuring drug product quality. This has been to some extent possible because of the availability of precise, rugged and reliable dissolution test equipment and increased knowledge and understanding of the science behind the test methodology. The in vitro dissolution/in vitro drug release is increasingly relied upon to assure product performance and product quality. The in vitro test procedure is a simple and economical method that can be utilized effectively in developing countries to assure acceptable drug product quality.

The engineering of dissolution test equipment has evolved over the years. Hence, the precision of current dissolution equipment calls into question the need for the 'calibrator tablets' and suggests that mechanical calibration of the unit may suffice. This seems to be consistent when looking at the global application of the dissolution test. The identification of "the right" calibrator tablet has become a major challenge these days, and it seems quite appropriate to switch over to mechanical calibration, especially considering the global usage of the dissolution test.

In recent years, the application of dissolution testing, also referred to as "in vitro release testing" has widened to include a variety of novel or special dosage forms including suspensions, semisolid topical preparations such as creams, ointments and gels, transdermal patches and suppositories. The drug release principles are also applied to chewing gums, implants, injectable microparticulate formulations and other newly developed technological preparations. The test is used in product development, in biopharmaceutics characterization of the drug product and as a quality control tool to assure consistent product performance.

As new chemical entities and new drug delivery systems are developed, we are challenged to develop new methods for in vitro dissolution / release rate determination. 


\section{Future Directions for Dissolution Testing in the Pharmaceutical Industry}

\author{
Vivian Gray \\ Dissolution Technologies, Hockessin, DE
}

$\mathrm{T}$ his article is a composite of comments from several Dissolution Technologies Editorial Board members who are currently active in the industry. We all seem to agree that we can't remember any dosage form test which has generated such rapid growth, interest and controversy in the field of pharmaceutical research. However, there are several aspects of the test that have changed and will continue to change.

We expect to see more development and application of automated methods at earlier stages of R\&D development. There will be more research into "novel" or "alternative" in vitro test systems that may better predict in vivo performance of drug products. This exploration is required to meet the needs of newer unique dosage forms that utilize innovative ways to administer the drugs to the patient, for example, implants, stents, microspheres and other modified release products. Contemporary devices such as USP Apparatus 4 and USP Apparatus 7, including newer modifications of these official apparatus, have shown themselves to be of great value in testing these novel dosage forms. The literature is starting to show many new devices and designs. There is even a resurrection and improvement of the older devices, such as the rotating bottle. There is potentially greater utilization of commercially-available pre-made dissolution media, especially for routine testing.

Over the past ten years much work has been done in the area of profile comparisons. The $\mathrm{f} 1$ and $\mathrm{f} 2$ metrics have proved to be useful to quantify similarity and difference between two profiles. However these formulae have limitations when dealing with full profile sampling. New mathematical tools may become necessary to replace $\mathrm{f} 1$ and $\mathrm{f} 2$ testing when one may generate dozens or hundreds of points in a profile. Multivariate analysis, mathematical formulae, and chemometrics could become the next area of growth in the reporting of dissolution results.

In addition to mathematical tools another area of growth has been in the classification and systemization of drug products. The available tools have given us a greater choice in investigating the properties of our dosage forms. Method development may begin to have more standardized strategies, for example, better alignment with the Biopharmaceutics Classification System, a more scientific approach to establishing "discriminating" conditions, and a better alignment of the establishment of the final method with drug development timelines, e.g., after formulation lock-in, prior to generating data used for final regulatory filings and establishing shelf life and specifications. A persistent challenge is the development of the appropriate testing methodology for poorly soluble drugs. The ideal method- ology would provide a good measure of manufacturing and process control along with a strong indication of bioavailability. There may be more attention paid to the formulation design and excipient matrix as well as the intrinsic dissolution properties of the drug substance.

We have seen that the possibility of alternative test methods such as disintegration and spectroscopy may be justified. The investigation of spectroscopic techniques to study possible correlations with conventional in vitro dissolution tests is ongoing and some predict that NIR may replace dissolution testing at the manufacturing site for online testing. More acceptance of fiber-optic dissolution systems, as more extensive applications are discovered (other than just dissolution) will probably occur.

In spite of the fact that many analysts in Quality Control consider dissolution testing to be merely a QC test, with little or no product performance applicability, many of us believe that it is highly predictive of the rate, and to some degree the extent, of drug absorption. In addition, we have seen the utilization of In Vitro and In Vivo Correlations (IVIVC) opening the doors for the predictive development of oral drug products, especially for ER systems. The use of IVIVC will expand in the future and allow us to use this physical test to anticipate the performance of a drug product possibly even to the extent of its correlation with active ingredient at the site clinical effect, as well as clinical effect itself.

Significant scientific, technical and regulatory work and effort has been invested over more than three decades, leading to state-of-the art testing methods and procedures and to a high level of standardization. In the future we see a continued growth and expansion of dissolution testing. Further effort is required to maintain and share this knowledge and experience and to make sure that the scientific background and empirical database, which supports the conclusions and concepts so far, will be maintained, further built and updated. It is important that young scientists make their own experience and collect their own learnings however, turning in circles or re-inventing the wheel cannot be considered the most efficient approach. There is a strong need and obligation to communicate, to share new scientific and technical results in the area of in vitro dissolution. There is no less a need of a publication platform for conference or seminar reports, summarizing the current state of thinking of those who are drivers in the areas of academia, regulatory or industrial pharmacy. Even more important are publications about in vitro dissolution/release testing of novel dosage forms. There is a significant need in this area. Formulations have become so specific that the portfolio of test apparatus and conventional test methods will not suffice, 
and standardization or even description of innovative tests in Pharmacopeias is not to be expected.

On the regulatory front, the idea of patenting a dissolution curve to extend patent life may continue although there is some concern that this practice may interfere with the generic equivalency program. However, methods to achieve dissolution curves may be patentable, there does seem to be such procedures in patents today. This trend will probably continue.

We have seen many changes in the field of dissolution, and anticipate a future of continuing change. There is much to learn and many new and interesting testing methodologies will be developed. This is a time for innovation and creativity.

I would like to acknowledge Lew Leeson, Ruben Lozano, Peter Scott, and Martin Siewert for their thoughtful contributions to this article. 